\title{
CARACTERIZAÇÃO DA EXTRAÇÃO DE ETANOL DE SOLUÇÕES HIDROALCOÓLICAS POR ARRASTE COM $\mathrm{CO}_{2}$
}

\author{
J. L. S. SONEGO ${ }^{1}$, D. A. LEMOS $^{1}$, C. E. M. PINTO ${ }^{2}$, A. J. G. CRUZ ${ }^{1}$, A. C. BADINO ${ }^{1 *}$ \\ ${ }^{1}$ Universidade Federal de São Carlos, Programa de Pós-Graduação em Engenharia Química \\ ${ }^{2}$ Universidade Federal de São Carlos, Departamento de Engenharia Química \\ "e-mail: badinojr@ufscar.br
}

\begin{abstract}
RESUMO
No presente estudo foram avaliadas as principais variáveis envolvidas na remoção de etanol a partir a de uma solução hidroalcoólica empregando $\mathrm{CO}_{2}$ como gás de arraste. Para avaliar a influência das variáveis de processo no arraste foi realizado um planejamento composto central rotacional (DCCR), variando a vazão específica de $\mathrm{CO}_{2}$, a concentração inicial de etanol e a temperatura solução. Como variáveis de resposta para o planejamento experimental, foram obtidos os valores das constantes de velocidade de remoção de etanol $\left(\mathrm{k}_{\mathrm{E}}\right)$ e de água $\left(\mathrm{k}_{\mathrm{W}}\right)$. A partir da análise estatística do planejamento experimental foi possível obter o modelo empírico para as variáveis resposta $\mathrm{k}_{\mathrm{E}} \mathrm{e} \mathrm{k}_{\mathrm{W}}$ e as curvas de contorno em função das variáveis estudadas $\left(\phi, \mathrm{C}_{\mathrm{E}} \mathrm{e} \mathrm{T}\right)$. Os resultados indicam que a vazão específica de $\mathrm{CO}_{2}$ foi a variável que apresentou maior efeito sobre as respostas analisadas.
\end{abstract}

\section{INTRODUÇÃo}

Nos últimos anos, o impacto ambiental associado ao excessivo uso de produtos derivados do petróleo, em especial os combustíveis, tem levado a uma busca crescente por bioenergia. Neste cenário, o etanol por ser renovável e ambientalmente correto, apresenta-se como uma das melhores alternativas como fonte de energia (BAI; ANDERSON; MOO-YOUNG, 2008).

A produção de etanol no Brasil é baseada essencialmente na fermentação do açúcar da cana. O processo mais utilizado é o de batelada alimentada, realizado industrialmente em dornas de grandes volumes (500 a $3.000 \mathrm{~m}^{3}$ ), onde é empregada levedura Saccharomyces cerevisiae. Ao final das fermentações que duram entre 6 e 11 horas e temperatura entre 32 a $35^{\circ} \mathrm{C}$, a concentração de etanol no caldo atinge valores na faixa de $8-11 \%(\mathrm{v} / \mathrm{v})$ (AMORIM et al., 2011).

Durante o processo fermentativo industrial, alguns fatores podem afetar negativamente a produção de etanol, entre eles o etanol acumulado, a temperatura elevada, a elevada pressão osmótica devido à presença de açúcares e sais, bem como acidez e contaminação com sulfitos e bactérias (GIBSON et al., 2007).

Entre os fatores, o etanol é o principal componente tóxico para levedura, devido a sua ação como um inibidor não competitivo do metabolismo. Isso resulta em uma diminuição no crescimento das leveduras e da produção de etanol à medida que a concentração de etanol no caldo de fermentação aumenta (MAIORELLA; BLANCH; WILKE, 1983). O acúmulo de etanol em concentrações superiores a 40 g. $\mathrm{L}^{-1}$ diminui significativamente as velocidades de 
crescimento celular e de produção de etanol, reduzindo a produtividade do processo (AIBA; SHODA; NAGATANI, 1968).

Uma forma de contornar o efeito de inibição sobre a levedura e assim obter uma elevada produtividade seria realizar a remoção do etanol a partir do caldo de fermentação durante o processo (CARDONA; SÁNCHEZ， 2007; SCHÜGERL， 2000; TAYLOR et al., 1995). Para remover o etanol, técnicas de separação podem ser empregadas como, a extração a vácuo, a pervaporação, a extração líquido-líquido, adsorção ou arraste gasoso. Esta última técnica é atraente para a produção em larga escala, devido a sua relativa simplicidade, à capacidade de remover os compostos voláteis e ainda possibilitar a utilização de um gás de extração de baixo custo como o dióxido de carbono gerado no próprio processo (ABREU-CAVALHEIRO; MONTEIRO, 2013).

Num trabalho recente, Sonego et al. (2014) estudaram a produção de etanol a partir de sacarose de cana-de-açúcar (180 g. $\mathrm{L}^{-1}$ ) utilizando fermentação extrativa em batelada empregando $\mathrm{CO}_{2}$ como gás de arraste. Foi demonstrado que o efeito de inibição do etanol sobre a levedura foi reduzido, resultando em aumento da produtividade de etanol em torno de $25,0 \%$ em relação à fermentação convencional, sem a remoção do etanol.

Assim, o estudo das variáveis envolvidas na remoção de etanol por arraste com $\mathrm{CO}_{2}$ torna-se necessário para proporcionar melhorias no desempenho do processo de esgotamento por $\mathrm{CO}_{2}$. Nesse contexto, o objetivo deste trabalho foi avaliar as influências da vazão específica de $\mathrm{CO}_{2}(\phi)$, concentração inicial de etanol $\left(\mathrm{C}_{\mathrm{E}}\right)$ e temperatura da solução (T) durante o arraste de etanol por $\mathrm{CO}_{2}$ a partir de uma solução hidroalcoólica.

\section{MATERIAIS E MÉTODOS}

Com a finalidade de avaliar quais variáveis apresentam maior influência no processo de esgotamento com dióxido de carbono foi realizado um estudo do arraste de etanol a partir de uma solução hidroalcoólica, variando a vazão específica de alimentação de $\mathrm{CO}_{2}(\phi)$, a concentração de etanol $\left(\mathrm{C}_{\mathrm{E}}\right)$ e a temperatura da solução $(\mathrm{T})$.

\subsection{Equipamento empregado nos experimentos}

Os experimentos para estudo do arraste foram conduzidos em reator pneumático tipo coluna de bolhas encamisado com volume útil de 5 L (BADINO; CERRI; HOKKA, 2007). Foi utilizada uma solução hidroalcoólica com concentração inicial de etanol variando de acordo com o planejamento experimental e o gás de arraste foi dióxido de carbono comercial armazenado em torpedo. Para controlar a vazão de gás foi empregado um fluxômetro de massa AALBORG, modelo GFC 37. A temperatura do reator foi mantida utilizando banho termostatizado. Durante a realização dos experimentos foi usado um termômetro digital marca Hanna Instruments, modelo HI 147-00 para monitorar a temperatura da solução. Foram retiradas amostras a cada $1 \mathrm{~h}$ durante $6 \mathrm{~h}$ de esgotamento, para determinação da concentração de etanol.

\subsection{Caracterizações da remoção de etanol por arraste com $\mathrm{CO}_{2}$}

Para o estudo das variáveis mais importantes no processo de arraste gasoso do etanol foi realizado um Delineamento Composto Central Rotacional com dois níveis, utilizando o software Statistica 7.0, tendo $2^{3}$ pontos fatoriais, 6 pontos axiais e 3 repetições no ponto central, totalizando 17 
experimentos (RODRIGUES E IEMMA, 2009).

Os níveis $(-1,68,-1,0,+1,+1,68)$ das variáveis independentes utilizadas no planejamento foram 1,$0 ; 1,6 ; 2,5 ; 3,4$ e $4 \mathrm{vvm}$ para a variável vazão específica de gás $(\phi)$, 30,$0 ; 36,1 ; 53,9$ e 60 g.L L $^{-1}$ para a concentração inicial de etanol $\left(\mathrm{C}_{\mathrm{E}}\right)$ e $30,30,8,32,33,2$ e 34 ${ }^{\circ} \mathrm{C}$ para temperatura da solução $(\mathrm{T})$.

A Tabela 1 mostra a matriz com os valores utilizados no planejamento experimental para as três variáveis independentes analisadas.

Tabela 1 - Matriz do planejamento fatorial

\begin{tabular}{cccc}
\hline Ensaio & $\mathbf{X}_{\mathbf{1}}(\phi)$ & $\mathbf{X}_{\mathbf{2}}\left(\mathrm{C}_{\mathrm{E}}\right)$ & $\mathbf{X}_{\mathbf{3}}(\mathrm{T})$ \\
\hline 1 & $-1(1,6)$ & $-1(36,1)$ & $-1(30,8)$ \\
2 & $-1(1,6)$ & $-1(36,1)$ & $1(33,2)$ \\
3 & $-1(1,6)$ & $1(53,9)$ & $-1(30,8)$ \\
4 & $-1(1,6)$ & $1(53,9)$ & $1(33,2)$ \\
5 & $1(3,4)$ & $-1(36,1)$ & $-1(30,8)$ \\
6 & $1(3,4)$ & $-1(36,1)$ & $1(33,2)$ \\
7 & $1(3,4)$ & $1(53,9)$ & $-1(30,8)$ \\
8 & $1(3,4)$ & $1(53,9)$ & $1(33,2)$ \\
9 & $-1,68(1)$ & $0(45)$ & $0(32)$ \\
10 & $1,68(4)$ & $0(45)$ & $0(32)$ \\
11 & $0(2,5)$ & $-1,68(30)$ & $0(32)$ \\
12 & $0(2,5)$ & $1,68(60)$ & $0(32)$ \\
13 & $0(2,5)$ & $0(45)$ & $-1,68(30)$ \\
14 & $0(2,5)$ & $0(45)$ & $1,68(34)$ \\
15 & $0(2,5)$ & $0(45)$ & $0(32)$ \\
16 & $0(2,5)$ & $0(45)$ & $0(32)$ \\
17 & $0(2,5)$ & $0(45)$ & $0(32)$ \\
\hline
\end{tabular}

Fonte: Autor (2015)

Como variáveis resposta do planejamento foram obtidos os valores das constantes de velocidades de remoção de etanol $\left(\mathrm{k}_{\mathrm{E}}\right)$ e de água $\left(\mathrm{k}_{\mathrm{W}}\right)$.

O arraste de etanol a partir de uma solução hidroalcoólica apresenta o mesmo comportamento que outros solventes orgânicos, podendo a velocidade de remoção ser expressa por um modelo de primeira ordem em relação à concentração de etanol
$\left(\mathrm{C}_{\mathrm{E}}\right)$ (DE VRIJE et al., 2013; SONEGO et al., 2014) de acordo com a Equação 1. Como durante os experimentos de arraste tem-se a remoção de água, a velocidade de remoção de água pode ser expressa pela Equação 2.

$$
\begin{aligned}
& \mathrm{R}_{\mathrm{E}}=-\frac{\mathrm{dC}_{\mathrm{E}}}{\mathrm{dt}}=\mathrm{k}_{\mathrm{E}} \cdot \mathrm{C}_{\mathrm{E}} \\
& \mathrm{R}_{\mathrm{W}}=\frac{\mathrm{dC}_{\mathrm{W}}}{\mathrm{dt}}=\mathrm{k}_{\mathrm{W}} \cdot \mathrm{C}_{\mathrm{W}}
\end{aligned}
$$

onde $R_{E}$ é a velocidade de remoção de etanol $\left(\mathrm{g} \cdot \mathrm{L}^{-1} \cdot \mathrm{h}^{-1}\right), \mathrm{R}_{\mathrm{W}}$ é a velocidade de remoção de água $\left(\mathrm{g} \cdot \mathrm{L}^{-1} \cdot \mathrm{h}^{-1}\right), \mathrm{C}_{\mathrm{E}}$ é a concentração de etanol na fase líquida $\left(\mathrm{g} . \mathrm{L}^{-1}\right)$ e $\mathrm{k}_{\mathrm{E}}$ é constante de velocidade de remoção de etanol $\left(\mathrm{h}^{-1}\right), \mathrm{C}_{\mathrm{W}}$ é a concentração de água na solução (g.L $\left.\mathrm{L}^{-1}\right)$ e $\mathrm{k}_{\mathrm{W}}$ é constante da velocidade de remoção de água $\left(\mathrm{h}^{-1}\right)$.

As constantes de velocidade de remoção de etanol e água $\left(\mathrm{k}_{\mathrm{E}} \mathrm{e} \mathrm{k}_{\mathrm{W}}\right)$ foram determinadas a partir do ajuste das curvas exponenciais dadas pelas Equações 3 e 4 aos dados experimentais de $\mathrm{C}_{\mathrm{E}}$ e $\mathrm{C}_{\mathrm{W}}$ ao longo do tempo (t), obtidos a partir do planejamento experimental.

$$
\begin{aligned}
C_{E} & =C_{E 0} \cdot e^{-k_{E} \cdot t} \\
C_{W} & =C_{W 0} \cdot e^{k_{W} \cdot t}
\end{aligned}
$$

onde $\mathrm{C}_{\mathrm{E} 0}$ e $\mathrm{C}_{\mathrm{W} 0}$ são as concentrações iniciais de etanol e de água na solução hidroalcoólica (g.L $\left.{ }^{-1}\right)$.

\subsection{Determinação da concentração de etanol}

A concentração de etanol foi determinada empregando HPLC (Waters), equipado com detector de índice de refração. Foi utilizada coluna Sugar-Pak I $(300$ x 6,5 $\mathrm{mm}, 10 \mu \mathrm{m}$, Waters) operada a $80^{\circ} \mathrm{C}$. Água ultrapura foi usada como eluente a uma vazão de $0,5 \mathrm{~mL} \cdot \mathrm{min}^{-1}$. Como padrão foi empregada 
solução de etanol com concentrações de 0,1 a 8 g.L L $^{-1}$ (SONEGO et al., 2014).

\section{RESULTADOS E DISCUSSÃO}

O emprego da ferramenta de planejamento experimental e análise de superfície de resposta possibilitou investigar a influência das variáveis $\phi, \mathrm{C}_{\mathrm{E} 0}$ e $\mathrm{T}$ no processo de remoção de etanol por $\mathrm{CO}_{2}$.

$\mathrm{Na}$ Tabela 2 são mostrados os resultados experimentais das constantes de velocidade de remoção de etanol e de água $\left(\mathrm{k}_{\mathrm{E}}\right.$ e $\left.\mathrm{k}_{\mathrm{W}}\right)$ para diferentes valores de vazão específica de gás $(\phi)$, concentração de etanol $\left(\mathrm{C}_{\mathrm{E}}\right)$ e para temperatura da solução $(\mathrm{T})$.

Tabela 2 - Resultados experimentais de $\mathrm{k}_{\mathrm{E}} \mathrm{e} \mathrm{k}_{\mathrm{w}}$

\begin{tabular}{ccc}
\hline Ensaio & $\mathrm{k}_{\mathrm{E}}\left(\mathrm{h}^{-1}\right)$ & $\mathrm{k}_{\mathrm{W}}\left(\mathrm{h}^{-1}\right)$ \\
\hline 1 & 0,0329 & 0,00135 \\
2 & 0,0335 & 0,00136 \\
3 & 0,0333 & 0,00210 \\
4 & 0,0377 & 0,00236 \\
5 & 0,0757 & 0,00292 \\
6 & 0,0841 & 0,00308 \\
7 & 0,0766 & 0,00446 \\
8 & 0,0911 & 0,00514 \\
9 & 0,0220 & 0,00119 \\
10 & 0,0962 & 0,00423 \\
11 & 0,0498 & 0,00162 \\
12 & 0,0692 & 0,00450 \\
13 & 0,0554 & 0,00275 \\
14 & 0,073 & 0,00362 \\
15 & 0,0573 & 0,00279 \\
16 & 0,0544 & 0,00261 \\
17 & 0,0540 & 0,00266 \\
\hline
\end{tabular}

Fonte: Autor (2015)

A partir dos valores de $\mathrm{k}_{\mathrm{E}}$ e $\mathrm{k}_{\mathrm{W}}$ (Tabela 2) verificou-se que os ensaios com as vazões de $\mathrm{CO}_{2}$ mais altas obtiveram os maiores valores de $\mathrm{k}_{\mathrm{E}}$, como pode ser visto nos ensaios $5 \quad\left(k_{\mathrm{E}}=0,0757 \quad \mathrm{~h}^{-1}\right), \quad 6 \quad\left(\mathrm{k}_{\mathrm{E}}=0,0841 \mathrm{~h}^{-1}\right), \quad 7$ $\left(k_{\mathrm{E}}=0,0766 \mathrm{~h}^{-1}\right), \quad 8 \quad\left(\mathrm{k}_{\mathrm{E}}=0,0911 \mathrm{~h}^{-1}\right)$ e 10
$\left(\mathrm{k}_{\mathrm{E}}=0,0962 \mathrm{~h}^{-1}\right)$. Os resultados indicam que valores de $\mathrm{k}_{\mathrm{E}}$ se elevam com o aumento da vazão específica de $\mathrm{CO}_{2}$ e da concentração inicial de etanol e com a diminuição da temperatura. Ademais, verificou-se que o ensaio conduzido com a vazão específica de $\mathrm{CO}_{2}$ mais elevada ( $\left.\phi=4 \mathrm{vvm}\right)$ acarretou no aumento da quantidade de água removida, resultando em um valor de $\mathrm{k}_{\mathrm{W}}=0,00423 \mathrm{~h}^{-1}$, observado no experimento 10 .

\subsection{Análise estatística do planejamento experimental - DCCR}

$\mathrm{Na}$ análise estatística do planejamento experimental foi adotado um nível de significância de 90\%, ou seja, foram considerados significativos os parâmetros em que $\mathrm{p}<0,1$. Com os resultados apresentados na Tabela 2 foi possível analisar estatisticamente o comportamento de cada resposta, empregando o software Statistica 7.0.

3.1.1 Análise dos resultados obtidos em termos da variável resposta $\mathrm{k}_{\mathrm{E}}$

A partir dos valores da Tabela 2, o comportamento das respostas $\mathrm{k}_{\mathrm{E}}$ foi analisado estatisticamente. Dessa forma, os coeficientes de regressão das variáveis e interações foram determinados, obtendo um modelo ajustado com as variáveis significativas codificadas, conforme a Equação 5.

$$
\begin{aligned}
Y_{k_{E}}= & 0,0569+0,0231 X_{1}+0,0033 X_{2}+ \\
& 0,0042 X_{3}+0,0021 X_{3}^{2}
\end{aligned}
$$

Observa-se pela Equação 5 uma maior influência da variável $X_{1}$ no aumento da constante de velocidade de remoção de etanol $\left(\mathrm{k}_{\mathrm{E}}\right)$, indicando que o emprego de elevadas vazões de alimentação de gás de arraste proporcionam aumentos na constante de velocidade de remoção de etanol. As variáveis 
$\mathrm{X}_{2}\left(\mathrm{C}_{\mathrm{E}}\right)$ e $\mathrm{X}_{3}(\mathrm{~T})$ também contribuem para o aumento do $\mathrm{k}_{\mathrm{E}}$, porém com um menor efeito.

$\mathrm{O}$ coeficiente de correlação $\left(\mathrm{R}^{2}\right)$ obtido após o ajuste foi de 0,98 , indicando um ajuste adequado aos dados experimentais do fator de arraste, mostrando que $98 \%$ da variabilidade dos dados foram explicados pela equação empírica proposta. A Tabela 3 apresenta o resultado da análise de variância (ANOVA) para o modelo.

Tabela 3 - Análise de variância para a constante de velocidade de remoção de etanol

\begin{tabular}{ccccc}
\hline $\begin{array}{c}\text { Fonte de } \\
\text { Variação }\end{array}$ & SQ & GL & QM & F $_{\text {calc. }}$ \\
\hline Regressão & 0,00771 & 4 & 0,00193 & 127,8 \\
Resíduos & 0,00018 & 12 & 0,000015 & \\
Total & 0,00789 & 16 & & \\
\hline
\end{tabular}

\% variação explicada $\left(\mathrm{R}^{2}\right)=98 ; \mathrm{F}_{\mathrm{tab} .(4,12,0,1)}=2,48$

Fonte: Autor (2015)

A ANOVA da Tabela 3 revela que o modelo que descreve a resposta constante de velocidade de remoção de etanol em função das variáveis investigadas com os parâmetros estatisticamente significativos, foi adequado. O F calculado foi aproximadamente quarenta e cinco vezes superior ao valor do F tabelado, o que satisfaz os requisitos para a construção das curvas de contorno.

A Figura 1 ilustra as curvas de contorno em função de $X_{1}(\phi)$ e $X_{2}\left(C_{E}\right)$ e $X_{3}(T)$ para a resposta $\mathrm{k}_{\mathrm{E}}$.

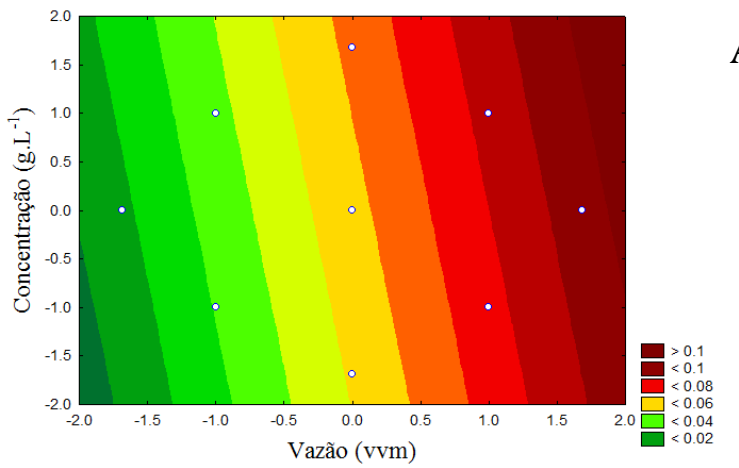

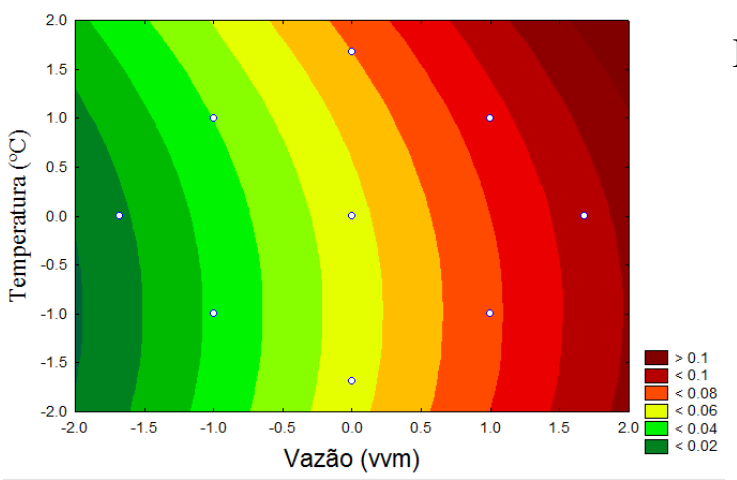

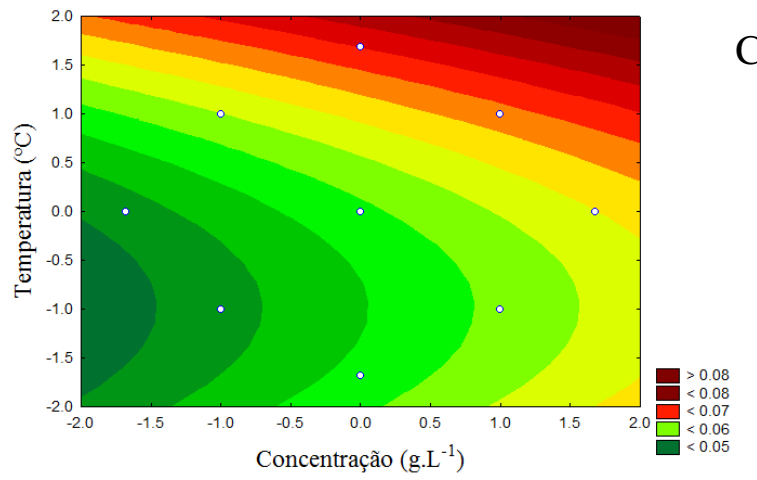

Figura 1 - Curvas de contorno para a resposta constante de velocidade de remoção de etanol $\left(\mathrm{k}_{\mathrm{E}}\right)$ : (A) função da vazão específica de $\mathrm{CO}_{2}(\phi)$ e concentração de inicial de etanol na solução $\left(\mathrm{C}_{\mathrm{E}}\right)$. (B) função da vazão específica de $\mathrm{CO}_{2}(\phi)$ e da temperatura da solução (T). (C) função da concentração de etanol $\left(\mathrm{C}_{\mathrm{E}}\right)$ e da temperatura da solução $(\mathrm{T})$.

A partir da Figura 1A observa-se que os maiores valores de $\mathrm{k}_{\mathrm{E}}$ foram obtidos nas faixas mais altas de vazão (3,4 a 4,0 vvm). Porém, a variável concentração de etanol na solução apresentou uma pequena influência na resposta constante de velocidade de remoção de etanol.

A Figura 1B ilustra a curva de contorno em função de $X_{1}$ e $X_{2}$ para o $k_{E}$, onde pode ser observado um aumento de $\mathrm{k}_{\mathrm{E}}$ em função do aumento da vazão específica de $\mathrm{CO}_{2}$.

Esse comportamento observado nas Figuras $1 \mathrm{~A}$ e $\mathrm{B}$ está relacionado à maior área superficial de bolhas disponível para arraste 
de etanol proporcionada quando altas vazões de $\mathrm{CO}_{2}$ foram empregadas. Esses resultados concordam com os obtidos por Sonego et al. (2014) ao estudar o arraste de etanol de uma solução hidroalcoólica.

A Figura 1C apresenta a curva de contorno em função de $X_{2}\left(C_{E}\right)$ e $X_{3}(T)$ para a resposta $\mathrm{k}_{\mathrm{E}}$. Pode-se observar que $\mathrm{o}$ aumento no valor de $k_{E}$ foi obtido para valores de concentração de etanol acima de 45 g. $L^{-1}$. A temperatura da solução hidroalcoólica $\left(34{ }^{\circ} \mathrm{C}\right)$ apresentou efeito positivo no $\mathrm{k}_{\mathrm{E}}$.

3.1.2 Análise dos resultados obtidos para a resposta $\mathrm{k}_{\mathrm{W}}$

A partir dos valores da Tabela 2, o comportamento da resposta $\mathrm{k}_{\mathrm{W}}$ foi analisada estatisticamente. Um modelo ajustado com as variáveis significativas codificadas foi obtido conforme o apresentado na Equação 6.

$$
\begin{aligned}
Y_{k_{w}}= & 0,002766+0,000992 X_{1}+0,000746 X_{2}+ \\
& 0,000188 X_{3}+0,000125 X_{3}^{2}+0,000231 X_{1} X_{2}
\end{aligned}
$$

A partir Equação 6 foi observado uma maior influência da variável $\mathrm{X}_{1}$ no aumento da constante de velocidade de remoção de água, indicando que elevadas vazões do gás de arraste proporcionam aumentos no $\mathrm{k}_{\mathrm{W}}$. As variáveis $\begin{array}{llllll}\mathrm{X}_{2} & \left(\mathrm{C}_{\mathrm{E}}\right) \text { e } & \mathrm{X}_{3} & (\mathrm{~T}) \text { também }\end{array}$ contribuem para o aumento do $\mathrm{k}_{\mathrm{W}}$, porém com um menor efeito.

O coeficiente de correlação $\left(\mathrm{R}^{2}\right)$ obtido após o ajuste foi de 0,97 , indicando um ajuste adequado aos dados experimentais do fator de arraste, mostrando que $97 \%$ da variabilidade dos dados foram explicados pela equação empírica proposta.

$O$ resultado da análise de variância (ANOVA) está apresentado na Tabela 4.
Tabela 4 - Análise de variância para a constante de velocidade de remoção de etanol

\begin{tabular}{ccccc}
\hline $\begin{array}{c}\text { Fontes de } \\
\text { Variação }\end{array}$ & SQ & GL & QM & $\mathrm{F}_{\text {calc }}$ \\
\hline Regressão & 0,000022 & 5 & 0,0000044 & 48 \\
Resíduos & 0,000001 & 11 & 0,00000009 & \\
Total & 0,000023 & 16 & & \\
\hline \% variação explicada $\left(\mathrm{R}^{2}\right)=97 ; \mathrm{F}_{\text {tab.(5,11,0,1) }}=2,45$ & \\
Fonte: Autor (2015)
\end{tabular}

A ANOVA da Tabela 4 revela que o modelo que descreve a resposta constante de velocidade de remoção de água $\left(\mathrm{k}_{\mathrm{W}}\right)$ em função das variáveis investigadas, com os parâmetros estatisticamente significativos, foi adequado. $\mathrm{O}$ valor de $\mathrm{F}$ calculado foi aproximadamente vinte vezes superior ao valor do $F$ tabelado, o que satisfaz os requisitos para a construção das curvas de contorno.

A Figura 2 ilustra as curvas de contorno em função de $X_{1}(\phi)$ e $X_{2}\left(C_{E}\right)$ para a resposta constante de velocidade de remoção de água $\left(\mathrm{k}_{\mathrm{W}}\right)$.

A partir da Figura 2A foi observado que os valores mais altos de vazão específica de $\mathrm{CO}_{2}(\sim 4,0 \mathrm{vvm})$ favorecem o arraste de água juntamente com o etanol. Assim, vazões de $\mathrm{CO}_{2}$ elevadas resultam em uma corrente na saída do reator diluída, em razão da maior quantidade de água.

Para uma remoção de etanol visando a redução do efeito de inibição, vazões de $\mathrm{CO}_{2}$ em torno de 2,5 vvm seriam mais vantajosas, proporcionando um arraste preferencial de etanol. 


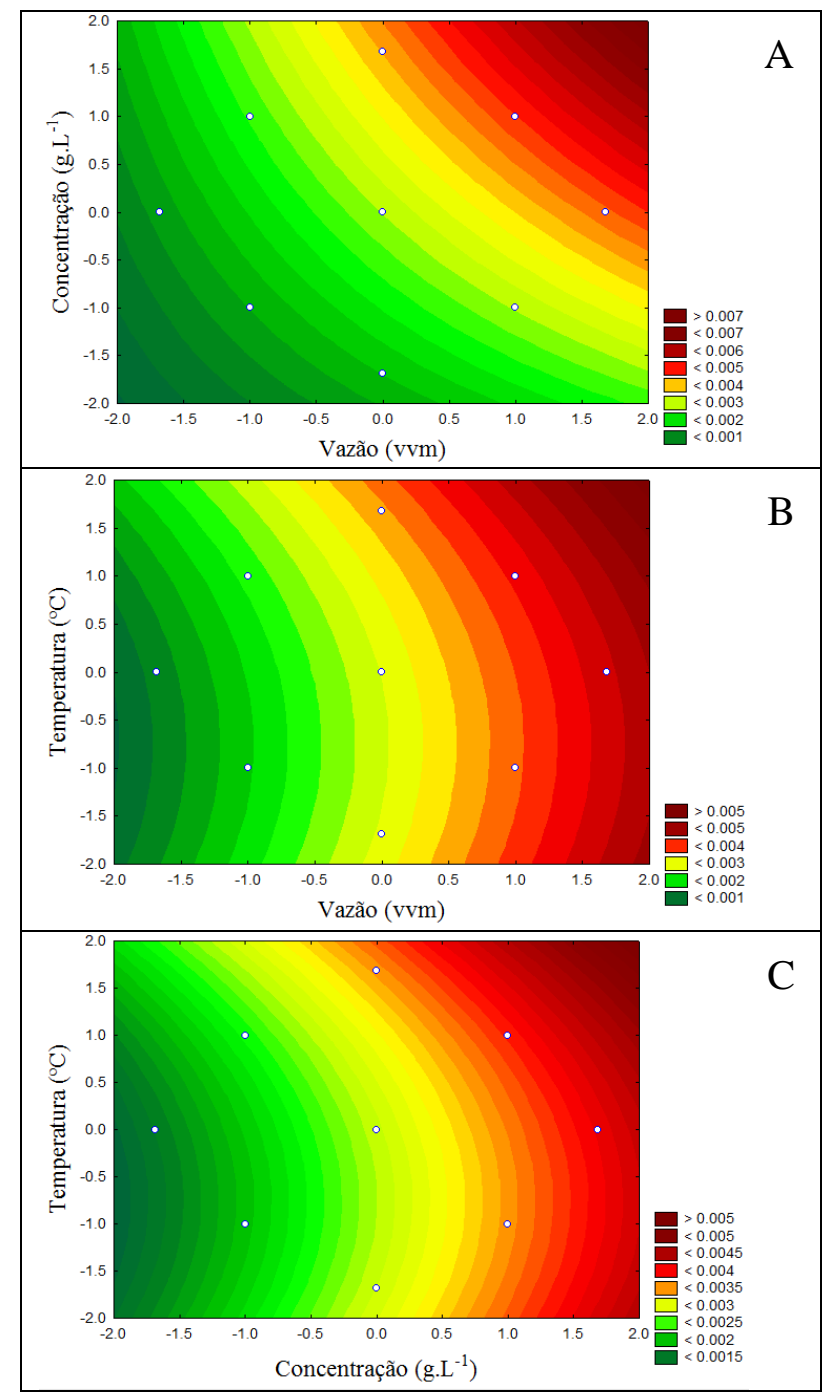

Figura 2 - Curvas de contorno para a resposta constante de velocidade de remoção de água $\left(\mathrm{k}_{\mathrm{W}}\right)$ : (A) função de $\phi$ e $\mathrm{C}_{\mathrm{E}}$. (B) função de $\phi$ e T. (C) função de $\mathrm{C}_{\mathrm{E}} \mathrm{e} \mathrm{T}$.

A Figura 2B ilustra a curva de contorno em função de $X_{1}(\phi)$ e $X_{2}\left(C_{E}\right)$ para o $k_{W}$, onde pode ser observado um aumento no valor dessa variável em função de $\phi$. Já a temperatura da solução apresentou pouca influência no valor de $\mathrm{k}_{\mathrm{W}}$.

A Figura 1C apresenta a curva de contorno em função de $\mathrm{X}_{2}\left(\mathrm{C}_{\mathrm{E}}\right)$ e $\mathrm{X}_{3}(\mathrm{~T})$ para a resposta $\mathrm{k}_{\mathrm{W}}$. Pode-se observar que $\mathrm{o}$ aumento no valor de $\mathrm{k}_{\mathrm{W}}$ foi obtido nos maiores valores de concentração de etanol (50 a 60 g.L $\mathrm{L}^{-1}$ ) e de temperatura da solução.

\section{CONCLUSÃO}

Os resultados obtidos neste estudo mostraram as influências das variáveis estudadas no processo de extração de etanol empregando arraste por $\mathrm{CO}_{2}$, possibilitando avaliar as condições experimentais que favoreçam obter um maior arraste de etanol. Os valores de $\mathrm{k}_{\mathrm{E}}$ e $\mathrm{k}_{\mathrm{W}}$ obtidos a partir do emprego do planejamento experimental indicaram que a vazão específica de $\mathrm{CO}_{2}$ foi a variável que mais influenciou no arraste de etanol, sendo o maior valor de $\mathrm{k}_{\mathrm{E}}\left(0,0962 \mathrm{~h}^{-1}\right)$ obtido no ensaio 10 , conduzido com a vazão específica de $\mathrm{CO}_{2}$ mais elevada ( $\left.\phi=4 \mathrm{vvm}\right)$. Com relação a quantidade de água removida, a vazão $\phi=4 \mathrm{vvm}$ acarretou no maior valor de $\mathrm{k}_{\mathrm{W}}\left(0,00423 \mathrm{~h}^{-1}\right)$.

\section{AGRADECIMENTOS}

Os autores agradecem a CAPES e à FAPESP (Proc. 2012/50046-4) pelos auxílios financeiros.

\section{REFERENCIAS}

ABREU-CAVALHEIRO, A.; MONTEIRO, G. Solving ethanol production problems with genetically modified yeast strainsBrazilian Journal of Microbiology, set. 2013.

AIBA, S.; SHODA, M.; NAGATANI, M. Kinetics of product inhibition in alcohol fermentation. Biotechnology and Bioengineering, v. 10, n. 6, p. 845-864, nov. 1968.

AMORIM, H. V; LOPES, M. L.; DE CASTRO OLIVEIRA, J. V.; BUCKERIDGE, M. S.; GOLDMAN, G. H. Scientific challenges of bioethanol production in Brazil. 
Applied microbiology and biotechnology, v. 91 , n. 5, p. 1267-75, set. 2011.

BADINO, A. C.; CERRI, M. O.; HOKKA, C. $O$. Sistema reacional pneumático e uso do mesmoBrazilian, 2007. Disponível em: $<$ http://www.patentesonline.com.br/sistemareacional-pneum-tico-e-uso-do-mesmo187759.html>

BAI, F. W.; ANDERSON, W. A.; MOOYOUNG, M. Ethanol fermentation technologies from sugar and starch feedstocks. Biotechnology advances, v. 26, n. 1, p. 89-105, 2008.

CARDONA, C. A.; SÁNCHEZ, O. J. Fuel ethanol production: process design trends and integration opportunities. Bioresource technology, v. 98, n. 12, p. 2415-57, set. 2007.

DE VRIJE, T.; BUDDE, M.; VAN DER WAL, H.; CLAASSEN, P. A. M.; LÓPEZCONTRERAS, A. M. "In situ" removal of isopropanol, butanol and ethanol from fermentation broth by gas stripping. Bioresource technology, v. 137, p. 153-9, jun. 2013.

GIBSON, B. R.; LAWRENCE, S. J.; LECLAIRE, J. P. R.; POWELL, C. D.; SMART, K. A. Yeast responses to stresses associated with industrial brewery handling. FEMS microbiology reviews, v. 31, n. 5, p. 535-69, set. 2007.

MAIORELLA, B.; BLANCH, H. W.; WILKE, C. R. By-product inhibition effects on ethanolic fermentation by Saccharomyces cerevisiae. Biotechnology and bioengineering, v. 25, n. 1, p. 103-21, jan. 1983.
RODRIGUES, M.I., IEMMA, A.F. Planejamento de Experimentos e Otimização de Processos: Uma estratégia Sequencial de planejamentos. Casa do Pão Editora, 2005.

SCHÜGERL, K. Integrated processing of biotechnology products. Biotechnology Advances, v. 18, n. 7, p. 581-599, nov. 2000.

SONEGO, J. L. S.; LEMOS, D. A.; RODRIGUEZ, G. Y.; CRUZ, A. J. G.; BADINO, A. C. Extractive Batch Fermentation with CO 2 Stripping for Ethanol Production in a Bubble Column Bioreactor: Experimental and Modeling. Energy \& Fuels, v. 28, n. 12, p. 7552-7559, 18 dez. 2014.

TAYLOR, F.; KURANTZ, M. J.; GOLDBERG, N.; CRAIG, J. C. Continuous fermentation and stripping of ethanol. Biotechnology progress, v. 11, n. 6, p. 6938, 1995. 